\title{
Supplementary Material: Reduction of Drug Toxicity Using Dendrimers Based on Melamine
}

Michael F. Neerman, Hui-Ting Chen, Alan R. Parrish and Eric E. Simanek

Animals. All animals were approved by the ULACC Committee, Texas A\&M University (AUP\#2003-68).

Preparation of Drug Solutions. Methotrexate (MTX) and 6-mercaptopurine (6-MP) were dissolved in $100 \mathrm{mM}$ saline and the $\mathrm{pH}$ was adjusted to 8 with $1 \mathrm{~N} \mathrm{NaOH}$ by adding base dropwise to obtain concentrations of $1.25 \mathrm{mg} / \mathrm{ml}$ and $2.5 \mathrm{mg} / \mathrm{ml}$, respectively. Dilutions of these stocks were prepared in saline to get final concentrations of $0.12 \mathrm{mg} / \mathrm{ml}$ and $0.21 \mathrm{mg} / \mathrm{ml}$ of MTX and 6-MP, respectively. Dendrimer was dissolved in saline at a concentration of $22.5 \mathrm{mg} / \mathrm{ml}$.

Preparations of Drug-Dendrimer Solutions. To solubilize the drugs with dendrimer, stock solutions of MTX and 6-MP were prepared in saline. A $50 \mathrm{mg}$ of MTX was dissolved in 40 $\mathrm{ml}$ of $100 \mathrm{mM}$ saline to get a concentration of $1.25 \mathrm{mg} / \mathrm{ml} .100 \mathrm{mg}$ of 6-MP was dissolved in 100 $\mathrm{mM}$ saline to get a concentration of $2.5 \mathrm{mg} / \mathrm{ml} 1.25 \mathrm{ml}$ of the MTX and $1.09 \mathrm{ml}$ of the 6-MP stocks were each added to $347 \mathrm{ul}$ of a dendrimer solution having a concentration of $22.5 \mathrm{mg} / \mathrm{ml}$. This solutions were sonicated for $5 \mathrm{~min}$. After such time, 11.4 and $11.56 \mathrm{ml}$ of saline were added to the MTX and 6-MP solutions, respectively, to yield a final working concentration of $.12 \mathrm{mg} / \mathrm{ml}$ MTX and .21 mg/ml 6-MP in .6 mg/ml dendrimer made up in a volume of $13 \mathrm{ml}$.

Synthesis of the Dendrimer. Solvents and reagents were reagent grade and used without further purification. ${ }^{1} \mathrm{H}$ NMR (300 MHz or $500 \mathrm{MHz}$ ) and proton decoupled ${ }^{13} \mathrm{C}$ NMR spectra (75 $\mathrm{MHz}$ or $125 \mathrm{MHz}$ ) were recorded with $\mathrm{CDCl}_{3}, \mathrm{CDCl}_{3} / \mathrm{MeOH}-\mathrm{d}_{4}(10: 1)$, or $\mathrm{MeOH}-\mathrm{d}_{4} / \mathrm{D}_{2} \mathrm{O}(2: 1)$ as internal standards. The synthetic scheme used is shown below:
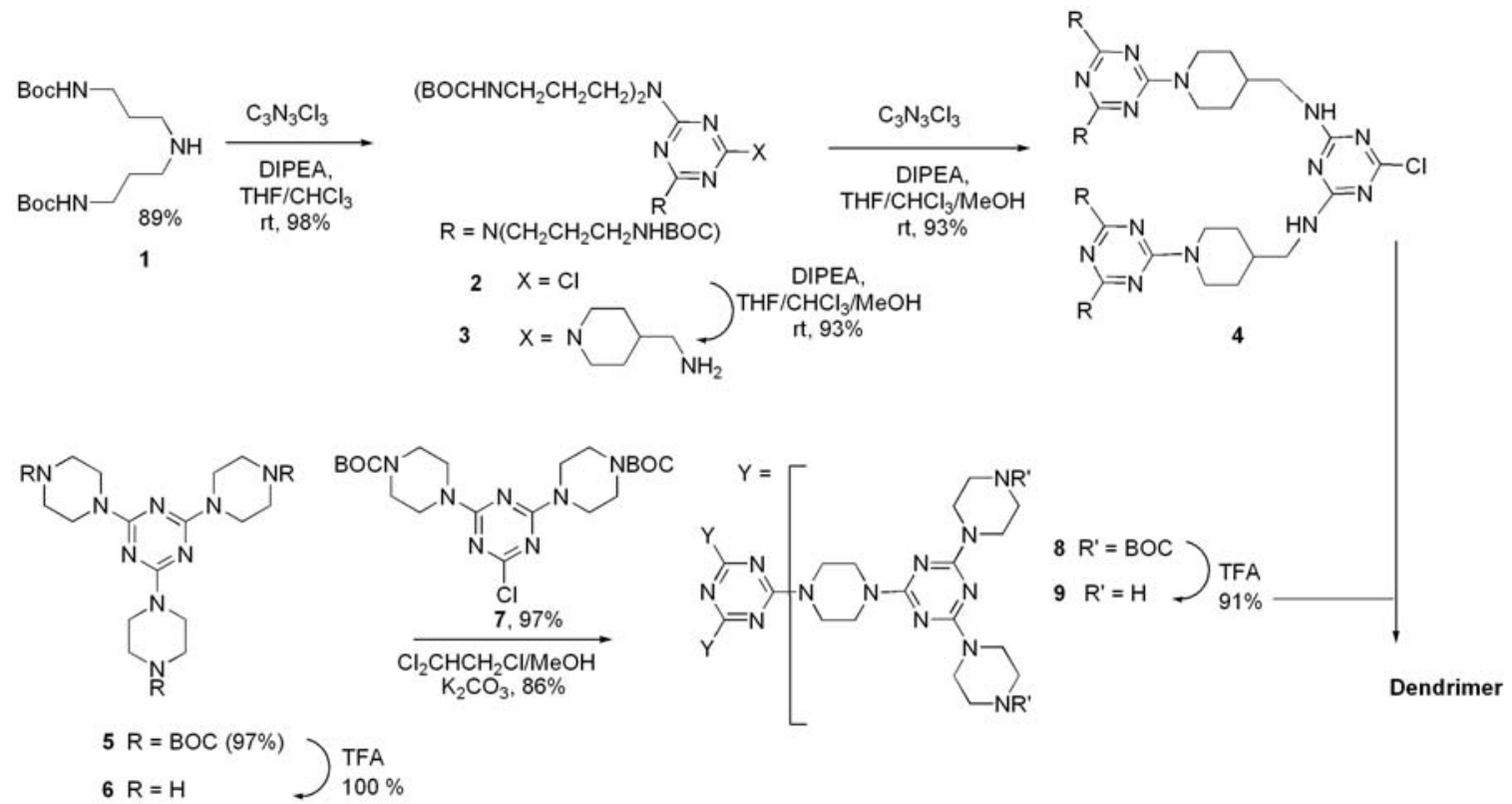

Intermediate 1. A solution of triamine (13.4 g, $0.1 \mathrm{~mol})$ and triethylamine (42.6 mL, $0.3 \mathrm{~mol})$ in THF $(500 \mathrm{~mL})$ was cooled in an ice bath and stirred as a solution of 2-[[tert-butoxycarbonyl]oxy]imino]-2-phenylacetonitrile (BOC-ON: $50 \mathrm{~g}, 0.2 \mathrm{~mol}$ ) in THF (500 mL) was added over $40 \mathrm{~min}$. After stirring for $2 \mathrm{hr}$ in the ice bath, the solution was warmed to room temperature 
and stirred overnight. The solvent was removed by evaporation to give golden oil which was dissolved in $\mathrm{CHCl}_{3}$ and washed with $5 \% \mathrm{HCl}$ solution (x3), $5 \% \mathrm{NaOH}(\mathrm{W} / \mathrm{V}$ ) solution (x4) and brine (x3). The organic layer was dried over anhydrous $\mathrm{MgSO}_{4}$, filtered, and the solvents were removed. The residue was purified by column chromatography $\left(\mathrm{MeOH} / \mathrm{CH}_{2} \mathrm{Cl}_{2}=1 / 10, \mathrm{R}_{f}=0.21\right)$ to provide 1. (29.4 g, $89 \%$ ). ${ }^{1} \mathrm{H}$ NMR $\left(\mathrm{CDCl}_{3}, 300 \mathrm{MHz}\right) \delta$ : 5.18 (brs, NH)), 3.20 (td, $J=6.0,6.3$ Hz, 4 H), 2.65 (t, J=6.6 Hz, 4 H), 1.69 (brs, NH), 1.65 (m, 4 H), 1.43 (s, 18H); ${ }^{13}$ C NMR $(\mathrm{CDCl} 3,75 \mathrm{~Hz}) \delta$ : 156.38, 79.27, 47.57, 39.09, 29.91, 28.64. MS (ESI-TOF): calcd for $\mathrm{C}_{16} \mathrm{H}_{33} \mathrm{~N}_{3} \mathrm{O}_{4}$ : 331.25; found $332.25(\mathrm{M}+\mathrm{H})^{+}$.

As per Westerberg, D. A.; Carney, P. L.; Rogers, P. E.; Kline, S. J.; Johnson, D. K. J. Med. Chem. 1989, 32, 236.

Intermediate 2. To an iced solution of 8 (10.0 g, $30.2 \mathrm{mmol})$ in $\mathrm{THF} / \mathrm{CHCl}_{3}(1: 1,50$ $\mathrm{mL})$, trichlorotriazine $(2.7 \mathrm{~g}, 14.4 \mathrm{mmol})$ and DIPEA $(10.7 \mathrm{~mL}, 60.0 \mathrm{mmol})$ were added. The mixture was warmed to room temperature and stirred for $2 \mathrm{hr}$. The white solid was removed by filtration, and the filtrate was evaporated to give an oil. The oil was dissolved in $\mathrm{CHCl}_{3}$ again and washed by $5 \% \mathrm{HCl}$ solution (x3), $5 \% \mathrm{NaOH}(\mathrm{W} / \mathrm{V})$ solution (x4) and brine (x3). The organic layer was dried over $\mathrm{MgSO}_{4}$, filtered and the solvent was removed to give 2 as foam. (10.9 g, 98 $\left.\%, \mathrm{MeOH} / \mathrm{CH}_{2} \mathrm{Cl}_{2}=1 / 20 \mathrm{R}_{\mathrm{f}}=0.25\right) .{ }^{1} \mathrm{H} \mathrm{NMR}\left(\mathrm{CDCl}_{3}, 300 \mathrm{MHz}\right) \delta: 5.65$ (brs, NH), 5.30 (brs, $\mathrm{NH}$ ), 3.60 (t, $J=6.0 \mathrm{~Hz}, 4 \mathrm{H}), 3.50$ (t, $J=7.2 \mathrm{~Hz}, 4 \mathrm{H}), 3.12$ (m, 8 H), 1.76 (m, 8 H), 1.45 (s, $18 \mathrm{H}), 1.44$ (s, $18 \mathrm{H}) ;{ }^{13} \mathrm{C} \mathrm{NMR}\left(\mathrm{CDCl}_{3}, 75 \mathrm{~Hz}\right) \delta: 169.34,165.11,156.66,79.69,79.28,45.15,44.07,38.74$, 37.22, 28.92, 28.89, 28.48. MS (ESI-TOF): calcd for $\mathrm{C}_{35} \mathrm{H}_{64} \mathrm{ClN}_{9} \mathrm{O}_{8}$ : 773.46; found 774.46 $(\mathrm{M}+\mathrm{H})^{+}$.

Intemediate 3. To the solution of 2 (9.8 g, $12.7 \mathrm{mmol})$ in $\mathrm{THF} / \mathrm{CHCl}_{3} / \mathrm{MeOH}(5: 5: 1$, $110 \mathrm{~mL}$ ) was added 4-(aminomethyl) piperidine (4.5 g, $29.0 \mathrm{mmol})$. The suspension was stirred at room temperature for $20 \mathrm{hr}$. A white solid was removed by filtration and the filtrate was evaporated to give oil. The oil was dissolved in $\mathrm{CHCl}_{3}$ again and washed by $5 \% \mathrm{HCl}$ solution (x3), $5 \% \mathrm{NaOH}(\mathrm{W} / \mathrm{V})$ solution (x4) and brine (x3). The organic layer was dried over $\mathrm{MgSO}_{4}$, filtered and condensed to afford foam as desired compound $3,\left(\mathrm{MeOH} / \mathrm{CH}_{2} \mathrm{Cl}_{2}=1 / 10, \mathrm{R}_{f}=0.13\right)$ yield 10.0 g. $(93 \%){ }^{1} \mathrm{H}$ NMR (MeOH- $\left.\mathrm{d}_{4}+\mathrm{CDCl}_{3}, 500 \mathrm{MHz}\right) \delta$ : 5.39 (brs, NH), 4.67 (d, $J=13.0$ Hz, 2H), 3.47 (br, 8 H), 3.05 (br, 8 H), 2.77 (t, J=12.3 Hz, 2 H), 2.56 (d, J=5.5 Hz, 2H), 1.69 (br, $10 \mathrm{H}), 1.53$ (br, $1 \mathrm{H}), 1.38$ (s, $36 \mathrm{H}), 1.09$ (m, $2 \mathrm{H}) ;{ }^{13} \mathrm{C} \mathrm{NMR}\left(\mathrm{CDCl}_{3}, 75 \mathrm{~Hz}\right) \delta: 165.31,164.71$, 155.95, 78.84, 43.26, 37.98, 37.37, 29.36, 28.39. MS (ESI-TOF): calcd for $\mathrm{C}_{41} \mathrm{H}_{77} \mathrm{~N}_{11} \mathrm{O}_{8}$ : 851.60; found $852.60(\mathrm{M}+\mathrm{H})^{+}$.

Intermediate 4. To the solution of $3(6.1 \mathrm{~g}, 7.2 \mathrm{mmol})$ in $\mathrm{THF} / \mathrm{CHCl}_{3} / \mathrm{MeOH}(5: 5: 1,110$ $\mathrm{mL}$ ) was added trichlorotriazine $(664.0 \mathrm{mg}, 3.6 \mathrm{mmol})$ and DIPEA $(2.6 \mathrm{~mL}, 14.3 \mathrm{mmol})$. The mixture was stirred at room temperature for $24 \mathrm{hr}$. After removing volatile components, the foam was purified through column chromatography to afford desired compound $\mathbf{1 1}$ as white foam. (6.0 g, 93 \%, EA/DCM=1/1, $\left.\mathrm{R}_{f}=0.33\right) .{ }^{1} \mathrm{H}$ NMR $\left(\mathrm{CDCl}_{3}+\mathrm{MeOH}-\mathrm{d}_{4}, 500 \mathrm{MHz}\right) \delta: 4.58(\mathrm{~m}, 4 \mathrm{H}), 3.39$ (brs, $16 \mathrm{H}), 3.17$ (m, $4 \mathrm{H}), 2.95$ (brs, $16 \mathrm{H}), 2.67$ (m, $4 \mathrm{H}), 1.62$ (m, $22 \mathrm{H}), 1.29$ (s, $72 \mathrm{H}), 1.06$ (m, $4 \mathrm{H}) ;{ }^{13} \mathrm{C}$ NMR $\left(\mathrm{CDCl}_{3}+\mathrm{MeOH}_{-} \mathrm{d}_{4}, 75 \mathrm{~Hz}\right) \delta: 165.19,164.65,156.25,79.02,45.98,43.00$, 42.58, 37.76, 36.50, 29.59, 28.15. MS (MALDI-TOF): calcd for $\mathrm{C}_{85} \mathrm{H}_{152} \mathrm{ClN}_{25} \mathrm{O}_{16}$ : 1815.73; found $1814.19(\mathrm{M})^{+}, 1836.73(\mathrm{M}+\mathrm{Na})^{+}$.

Intermediate 5. Boc-protected piperazine $(1.57 \mathrm{~g}, 8.3 \mathrm{mmol})$ and trichlorotriazine (0.5 g, $2.5 \mathrm{mmol})$ were mixed in $60 \mathrm{~mL}$ of THF/DMF (10/1). DIPEA (2.2 mL, $12.5 \mathrm{mmol})$ was added and the reaction was stirred at room temperature for $2 \mathrm{hr}$, then at $80^{\circ} \mathrm{C}$ for $5 \mathrm{hr}$. After being cooled to room temperature, a white solid was collected by filtration and washed with THF before drying under vacuum to yield compound $1.53 \mathrm{~g}$ of $5\left(97 \%, \mathrm{MeOH} / \mathrm{CH}_{2} \mathrm{Cl}_{2}=1 / 50, \mathrm{R}_{f}=0.26\right) .{ }^{1} \mathrm{H}$ NMR (CDCl $30300 \mathrm{MHz}) \delta: 3.74$ (m, $12 \mathrm{H}), 3.44$ (m, $12 \mathrm{H}), 1.47$ (s, $27 \mathrm{H}) ;{ }^{13} \mathrm{C} \mathrm{NMR}(\mathrm{CDCl} 3$, $75 \mathrm{~Hz}) \delta$ : 165.32, 154.83, 79.89, 42.90, 28.41. MS (ESI-TOF): calcd for $\mathrm{C}_{30} \mathrm{H}_{51} \mathrm{~N}_{9} \mathrm{O}_{6}$ : 633.40; found 634.40. $(\mathrm{M}+\mathrm{H})^{+}$. 
Intermediate 6. Intermediate 5 ( $2.1 \mathrm{~g}, 3.3 \mathrm{mmol}$ ) was dissolved in 20mL TFA/DCM (1:1). After $4 \mathrm{hr}$ stirring at room temperature, the volatile components were removed by evaporation. The residue was dissolved in $\mathrm{MeOH}$ and neutralized by adding trimethylamine, and the solvent was subsequently evaporated. After the procedure was repeated by three times, a

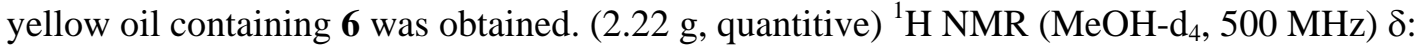

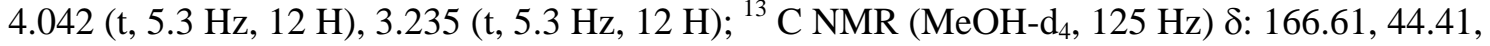
41.16. MS (ESI-TOF): cacld for $\mathrm{C}_{15} \mathrm{H}_{27} \mathrm{~N}_{9}$ : 333.24; found $334.25(\mathrm{M}+\mathrm{H})^{+}$.

Intermediate 7. Boc-protected piperazine (3.99 g, $21 \mathrm{mmol})$ and trichlorotriazine (1.86 g, $10.0 \mathrm{mmol})$ were mixed in THF $(100 \mathrm{~mL})$. DIPEA $(3.7 \mathrm{~mL}, 30.0 \mathrm{mmol})$ was added and the reaction was stirred at room temperature for $24 \mathrm{hr}$. A white solid was removed by filtration and the filtrate was evaporated to provide a residue. Following chromatography $\left(\mathrm{MeOH} / \mathrm{CH}_{2} \mathrm{Cl}_{2}=1 / 50, \mathrm{R}_{f}=0.36\right) 7$ was obtained as a white solid in $97 \%$ yield. ${ }^{1} \mathrm{H}$ NMR $\left(\mathrm{CDCl}_{3}\right.$, $300 \mathrm{MHz}$ ) $\delta$ : 3.755 (br s, $8 \mathrm{H}$ ), 3.444 (br s, $8 \mathrm{H}), 1.456$ (s, $\left.18 \mathrm{H}) ;{ }^{13} \mathrm{C} \mathrm{NMR} \mathrm{CDCl}_{3}, 75 \mathrm{~Hz}\right) \delta$ : 169.92, 164.69, 154.86, 80.48, 43.50, 28.61. MS (ESI-TOF): calcd for $\mathrm{C}_{21} \mathrm{H}_{34} \mathrm{ClN}_{7} \mathrm{O}_{4}$ : 483.24; found $484.25(\mathrm{M}+\mathrm{H})^{+}, 486.26(\mathrm{M}+\mathrm{H})^{+}$.

Intermediate 8. Intermediate $6(0.58 \mathrm{~g}, 1.74 \mathrm{mmol})$ was mixed with DIPEA $(1 \mathrm{~mL})$ in $20 \mathrm{~mL}$ of 1,1,2-trichloroethane/MeOH (1:1) for $5 \mathrm{~min}$. Intermediate 7 (3.0 g, $6.2 \mathrm{mmol}$ ) and $\mathrm{K}_{2} \mathrm{CO}_{3}(5.0 \mathrm{~g}, 36 \mathrm{mmol}$ ) were added to the mixture. The mixture was stirred under reflux for 24 hr. After cooling to room temperature, the solid was removed by filtration and washed with $\mathrm{CH}_{2} \mathrm{Cl}_{2}$ for several times. The filtrate was collected and evaporated to obtain solid. Purification by column chromatography ( $\left.\mathrm{MeOH} / \mathrm{CH}_{2} \mathrm{Cl}_{2}=1 / 20, \mathrm{R}_{f}=0.5\right)$ afforded 8 as a white solid ( $2.51 \mathrm{~g}, 86$

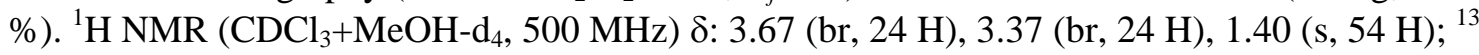
C NMR $\left(\mathrm{CDCl}_{3}+\mathrm{MeOH}_{-} \mathrm{d}_{4}, 125 \mathrm{~Hz}\right) \delta: 165.11,154.93,80.15$, 42.93, 42.8, 28.1. MS (MALDITOF): calcd for $\mathrm{C}_{78} \mathrm{H}_{126} \mathrm{~N}_{30} \mathrm{O}_{12}$ : 1676.03; found $1677.70(\mathrm{M}+\mathrm{H})^{+}$.

Intermediate 9. Intermediate 8 (1.86g, $1.1 \mathrm{mmol}$ ) was stirred in $\mathrm{CH}_{2} \mathrm{Cl}_{2} / \mathrm{MeOH}(15$ $\mathrm{mL} / 2 \mathrm{~mL})$. Trifluoroacetic acid (12 mL) was added to the iced solution. After stirring for $24 \mathrm{hr}$, the volatiles were evaporated to provide an oil. The oil was partitioned between $\mathrm{CHCl}_{3}$ and $5 \%$ $\mathrm{NaOH}(a q)$. The organic layer was washed by $5 \% \mathrm{NaOH}$ (x3) and brine (x3), and then dried over $\mathrm{MgSO}_{4}$. After filtering, the solvent was evaporated to afford $\mathbf{9}$ as a light yellow solid (1.08 g, 91\%). ${ }^{1} \mathrm{H}$ NMR ( $\left.\mathrm{CDCl}_{3}+\mathrm{MeOH}_{-} \mathrm{d}_{4}, 300 \mathrm{MHz}\right) \delta$ : 3.68 (br, $\left.12 \mathrm{H}\right), 3.63$ (m, $\left.12 \mathrm{H}\right), 2.73(\mathrm{~m}, 12 \mathrm{H})$; ${ }^{13} \mathrm{C}$ NMR $\left(\mathrm{CDCl}_{3}+\mathrm{MeOH}-\mathrm{d}_{4}, 75 \mathrm{~Hz}\right) \delta: 165.11,165.01,45.17,43.62$, 42.81. MS (ESI-TOF): calcd for $\mathrm{C}_{48} \mathrm{H}_{78} \mathrm{~N}_{30}$ : 1074.70; found $538.37(\mathrm{M}+2 \mathrm{H})^{2+}, 1075.75(\mathrm{M}+\mathrm{H})^{+}$.

Dendrimer. Intermediate 9 ( $0.54 \mathrm{~g}, 0.5 \mathrm{mmol})$ and polymer-supported BEMP (2.7 g, 6.0 $\mathrm{mml}$ ) were mixed in 1,1,2 trichloroethane/i-PrOH (5/1, $30 \mathrm{~mL}$ ) for $10 \mathrm{~min}$ at room temperature. To the mixture was added compound $4(5.54 \mathrm{~g}, 3.05 \mathrm{mmol})$ and the reaction temperature was raised to $80{ }^{\circ} \mathrm{C}$. After stirring for 3 days, the supported base was removed, the filtrate was evaporated and purified by column chromatography $\left(\mathrm{MeOH} / \mathrm{CH}_{2} \mathrm{Cl}_{2}=1 / 10, \mathrm{R}_{f}=0.35\right)$ to obtain Dendrimer (5.3 g, 90 \%). ${ }^{1} \mathrm{H} \mathrm{NMR}\left(\mathrm{MeOH}-\mathrm{d}_{4}+\mathrm{CDCl}_{3}, 500 \mathrm{MHz}\right) \delta$ : 5.63 (brs, NH), 5.34 (brs, $\mathrm{NH}$ ), 4.57 (d, J=8.5 Hz, $24 \mathrm{~Hz}$ ), 3.69 (br, 72 H), 3.37 (br, 96 H), 3.16 (br, 24 H), 2.94 (br, 96 H), 2.68 (br, $24 \mathrm{H}), 1.59$ (br, $132 \mathrm{H}), 1.28$ (s, $432 \mathrm{H}), 1.10$ (br, $24 \mathrm{H}) ;{ }^{13} \mathrm{C} \mathrm{NMR}\left(\mathrm{MeOH}_{-} \mathrm{d}_{4}+\mathrm{CDCl}_{3}\right.$, $125 \mathrm{MHz}$ ) $\delta:$ 165.07, 164.55, 156.19, 78.85, 45.77, 43.15, 42.96, 42.72, 42.47, 37.81, 37.02, 29.55, 28.05, 27.62. MS (MALDI-TOF): calcd for $\mathrm{C}_{558} \mathrm{H}_{984} \mathrm{~N}_{180} \mathrm{O}_{96}: 11750.9320$; found 5869.7 $(\mathrm{M}+2 \mathrm{H})^{2+}, 11764.54(\mathrm{M}+\mathrm{H})^{+}$.

Statistical Analysis. To calculate the standard deviation for each set of pooled data, the following equation was used: 


$$
s=\sqrt{\frac{\sum\left(x_{i}-\bar{x}\right)^{2}}{(n-1)}}
$$

Where $\mathbf{x}_{\mathbf{i}}=$ control value, $\mathbf{X}$-bar $=$ average, $\mathbf{n}=$ sample size

An example is performed from the G3/MTX treatment group:

$$
\begin{gathered}
\frac{(35-58.66)^{2}+(65.2-58.66)^{2}+(73-58.66)^{2}+(64.8-58.66)^{2}+(55.3-58.66)^{2}}{5-1} \\
\underline{214.297}=\quad \begin{array}{c}
\text { sqrt of } 53.57425 \\
4
\end{array}=\underline{\mathbf{1 4 . 6 3 8}}
\end{gathered}
$$

To calculate whether there existed significant differences between two means, the Student's t-test was used:

$$
t=\left|\bar{x}_{1}-\bar{x}_{2}\right| \div \sqrt{A * \bar{B}}
$$

\section{Where,}

$$
A=\left(n_{1}+n_{2}\right) \div n_{1} n_{2}
$$

\section{and}

$$
B=\left[\left(n_{1}-1\right) s_{1}^{2}+\left(n_{2}-1\right) s_{2}^{2}\right] \div\left[n_{1}+n_{2}-2\right]
$$

Where $\mathbf{X}$-bar is the sample mean, $\mathbf{s}$ is the sample standard deviation and $\mathbf{n}$ is the sample size

An example is performed using data from the controls and 6-MP treated groups to see if a significant difference is present between these two means:

$$
\begin{aligned}
& \mathbf{A}=(5+5) \div(5)(5)=.4 \\
& \mathbf{B}=\left[(5-1) 9.982^{2}+(5-1) 22.82^{2}\right] \div[5+5-2]=\mathbf{3 1 1 . 5 6 7} \\
& \text { So } \mathbf{t}=|51.6-88.68| \div \text { sqrt of }(.4)(311.567)=\mathbf{3 . 3 2 1 5}
\end{aligned}
$$

To intrepret this t value we use a probability table: 


\begin{tabular}{|c|c|c|c|c|}
\hline $\begin{array}{l}\text { Degrees of } \\
\text { Freedom }\end{array}$ & $\mathrm{p}=0.05$ & $\mathrm{p}=0.025$ & $\mathrm{p}=0.01$ & $\mathrm{p}=0.005$ \\
\hline $\begin{array}{l}1 \\
2 \\
3 \\
4 \\
5\end{array}$ & $\begin{array}{r}12.71 \\
4.30 \\
3.18 \\
2.78 \\
2.57\end{array}$ & $\begin{array}{r}25.45 \\
6.20 \\
4.17 \\
3.50 \\
3.16\end{array}$ & $\begin{array}{r}63.66 \\
9.92 \\
5.84 \\
4.60 \\
4.03\end{array}$ & $\begin{array}{r}127.32 \\
14.09 \\
7.45 \\
5.60 \\
4.77\end{array}$ \\
\hline $\begin{array}{r}6 \\
7 \\
8 \\
9 \\
10\end{array}$ & $\begin{array}{l}2.45 \\
2.36 \\
2.31 \\
2.26 \\
2.23\end{array}$ & $\begin{array}{l}2.97 \\
2.84 \\
2.75 \\
2.68 \\
2.63\end{array}$ & $\begin{array}{l}3.71 \\
3.50 \\
3.36 \\
3.25 \\
3.17\end{array}$ & $\begin{array}{l}4.32 \\
4.03 \\
3.83 \\
3.69 \\
3.58\end{array}$ \\
\hline $\begin{array}{l}11 \\
12 \\
13 \\
14 \\
15\end{array}$ & $\begin{array}{l}2.20 \\
2.18 \\
2.16 \\
2.14 \\
2.13\end{array}$ & $\begin{array}{l}2.59 \\
2.56 \\
2.53 \\
2.51 \\
2.49\end{array}$ & $\begin{array}{l}3.11 \\
3.05 \\
3.01 \\
2.98 \\
2.95\end{array}$ & $\begin{array}{l}3.50 \\
3.43 \\
3.37 \\
3.33 \\
3.29\end{array}$ \\
\hline $\begin{array}{l}16 \\
17 \\
18 \\
19 \\
20\end{array}$ & $\begin{array}{l}2.12 \\
2.11 \\
2.10 \\
2.09 \\
2.09\end{array}$ & $\begin{array}{l}2.47 \\
2.46 \\
2.44 \\
2.43 \\
2.42\end{array}$ & $\begin{array}{l}2.92 \\
2.90 \\
2.88 \\
2.86 \\
2.84\end{array}$ & $\begin{array}{l}3.25 \\
3.22 \\
3.20 \\
3.17 \\
3.15\end{array}$ \\
\hline $\begin{array}{l}21 \\
22 \\
23 \\
24 \\
25\end{array}$ & $\begin{array}{l}2.08 \\
2.07 \\
2.07 \\
2.06 \\
2.06\end{array}$ & $\begin{array}{l}2.41 \\
2.41 \\
2.40 \\
2.39 \\
2.38\end{array}$ & $\begin{array}{l}2.83 \\
2.82 \\
2.81 \\
2.80 \\
2.79\end{array}$ & $\begin{array}{l}3.14 \\
3.12 \\
3.10 \\
3.09 \\
3.08\end{array}$ \\
\hline $\begin{array}{l}26 \\
27 \\
28 \\
29 \\
30\end{array}$ & $\begin{array}{l}2.06 \\
2.05 \\
2.05 \\
2.04 \\
2.04\end{array}$ & $\begin{array}{l}2.38 \\
2.37 \\
2.37 \\
2.36 \\
2.36\end{array}$ & $\begin{array}{l}2.78 \\
2.77 \\
2.76 \\
2.76 \\
2.75\end{array}$ & $\begin{array}{l}3.07 \\
3.06 \\
3.05 \\
3.04 \\
3.03\end{array}$ \\
\hline $\begin{array}{l}40 \\
60 \\
120 \\
\text { infinity }\end{array}$ & $\begin{array}{l}2.02 \\
2.00 \\
1.98 \\
1.96\end{array}$ & $\begin{array}{l}2.33 \\
2.30 \\
2.27 \\
2.24\end{array}$ & $\begin{array}{l}2.70 \\
2.66 \\
2.62 \\
2.58\end{array}$ & $\begin{array}{l}2.97 \\
2.92 \\
2.86 \\
2.81\end{array}$ \\
\hline
\end{tabular}

We determine the degrees of freedom by the number of data points in the two groups combined minus 2. So,

$10-2=8$.

At 8 degrees of freedom we have a value of 2.31 at $p=0.05$. If $\mathbf{t}$ exceeds the tabled value of $p$ value (0.05), the means are significantly different at the probability level that is listed. Since our $\mathrm{t}$ value, 3.3215 is higher than 2.31, our means are significant different. 